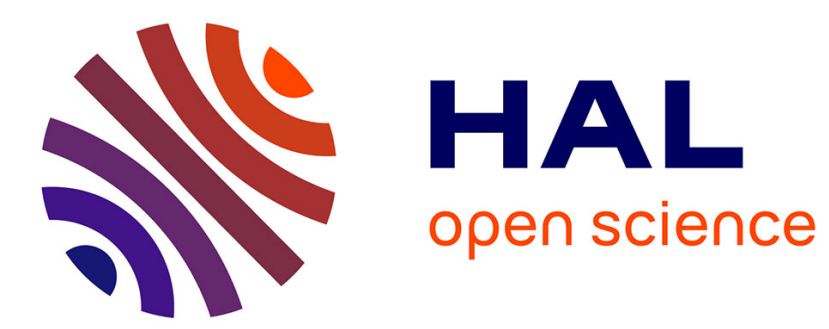

\title{
DIFFUSION QUASI ÉLASTIQUE
}

\author{
J.-R. Pizzi
}

\section{To cite this version:}

J.-R. Pizzi. DIFFUSION QUASI ÉLASTIQUE. Colloquium on Nuclear Surface, 1975, Dijon, France. pp.C5-63-C5-67, 10.1051/jphyscol:1975509 。 jpa-00216364

\section{HAL Id: jpa-00216364 https://hal.science/jpa-00216364}

Submitted on 1 Jan 1975

HAL is a multi-disciplinary open access archive for the deposit and dissemination of scientific research documents, whether they are published or not. The documents may come from teaching and research institutions in France or abroad, or from public or private research centers.
L'archive ouverte pluridisciplinaire HAL, est destinée au dépôt et à la diffusion de documents scientifiques de niveau recherche, publiés ou non, émanant des établissements d'enseignement et de recherche français ou étrangers, des laboratoires publics ou privés. 


\title{
DIFFUSION QUASI ÉLASTIQUE
}

\author{
J. R. PIZZI \\ Institut de Physique Nucléaire, Université Claude-Bernard, Lyon-1 \\ et IN2 P3, 43, bd du 11-Novembre-1918, 69621 Villeurbanne, France
}

\begin{abstract}
Résumé. - Dans une première partie, on rappelle les conditions cinématiques dans lesquelles sont étudiées les réactions de diffusion quasi élastique ainsi que l'approximation de l'impulsion qui est utilisée pour leur interprétation. On analyse ensuite dans quel sens ces expériences ont évolué dans les dernières années' Trois expériences récentes sont présentées et discutées. Deux concernent les sous-structures $\alpha$ étudiées par réaction $(\mathrm{p}, \mathrm{p} \alpha)$ à $157 \mathrm{MeV}$ et $600 \mathrm{MeV}$. La dernière s'intéresse aux agrégats $\mathrm{d}, \mathrm{t}$ et ${ }^{3} \mathrm{He}$ obtenus par réaction $(\mathrm{p}, \mathrm{p} x)$ à $75 \mathrm{MeV}$.
\end{abstract}

\begin{abstract}
In a first part, the kinematical conditions which are chosen to study quasi free scattering reactions are presented, as well as the impulse approximation which is used to interpret the experimental data. Then, the evolution of the study of these reactions in the last few years is analysed. Three recent experiments are presented and discussed. Two of them deal with $\alpha$-clusters studied by $(p, p \alpha)$ reaction at 157 and $600 \mathrm{MeV}$. The third is concerned with $\mathrm{d}, \mathrm{t}$ and ${ }^{3} \mathrm{He}$ clusters studied by $(\mathrm{p}, \mathrm{p} x)$ reaction at $75 \mathrm{MeV}$.
\end{abstract}

1. Introduction. - L'étude expérimentale des réactions de diffusion quasi élastique a toujours constitué une voie privilégiée pour la mise en évidence et l'étude d'agrégats dans les noyaux. Depuis les premières expériences sur ${ }^{6} \mathrm{Li}$ et ${ }^{7} \mathrm{Li}$ faites à Orsay [1], sur ${ }^{12} \mathrm{C}$ par James et Pugh en 1963 [2] et Igo et al. [3] la même année en $(\alpha, 2 \alpha)$ à $915 \mathrm{MeV}$, de nombreux résultats ont été publiés généralement pour des énergies incidentes relativement basses $(<100 \mathrm{MeV})$. Pour des raisons techniques, les protons et les alphas ont été utilisés comme particules incidentes et les efforts des expérimentateurs ont surtout porté sur l'analyse de la structure en agrégats $\alpha$ des noyaux. Pour mieux comprendre ce que l'on peut extraire comme renseignements de telles expériences, il n'est pas inutile de rappeler, brièvement, la cinématique qui régit ces réactions.

2. Conditions cinématiques et approximation de l'impulsion. - Dans une réaction de diffusion quasi élastique, une particule incidente, d'impulsion $\mathbf{p}_{0}$ interagit avec un noyau $N$. Dans la voie de sortie on retrouve la particule incidente avec une impulsion $\mathbf{p}_{1}$, un fragment $x$ du noyau avec une impulsion $\mathbf{p}_{2}$ et un noyau résiduel $\mathbf{C}$ avec une impulsion $\mathbf{p}_{\mathrm{R}}$. $\mathbf{L a}$ conservation de l'énergie et de l'impulsion s'écrit :

$$
\begin{aligned}
& E_{\mathrm{s}}=E_{0}-E_{1}-E_{2}-E_{\mathrm{R}} \\
& \mathbf{p}_{\mathrm{R}}=\mathbf{p}_{0}-\mathbf{p}_{1}-\mathbf{p}_{2}
\end{aligned}
$$

où $E_{\mathrm{s}}$ représente l'énergie de séparation de l'agrégat $x$.

Notons que ces équations de conservation s'appliquent à n'importe quelle réaction donnant trois corps dans la voie de sortie comme les réactions séquentielles, les réactions de cassure du noyau, etc... Il en résulte qu'il est souvent difficile de séparer les réactions quasi élastiques qui nous intéressent de celles-ci.

$\mathrm{Si}$ l'on appelle q l'impulsion relative entre les agrégats $x$ et $C$ dans le noyau avant le choc, l'approximation de l'impulsion s'exprimera en écrivant que $\mathbf{q}=-\mathbf{p}_{\mathrm{R}}$. La figure 1 schématise une telle réaction et le graphe bien connu qui lui correspond.

Dans cette approximation, la section efficace prend la forme :

$$
\frac{\mathrm{d}^{3} \sigma}{\mathrm{d} \Omega_{1} \mathrm{~d} \Omega_{2} \mathrm{~d} E}=\mu S|g(q)|^{2}\left(\frac{\mathrm{d} \sigma}{\mathrm{d} \Omega}\right)_{0, x}
$$

$\mu$ est un facteur cinématique. En ondes planes (PWIA), $g(q)$ est la transformée de Fourier de la fonction d'onde qui décrit le mouvement relatif de $x$ et de C. En ondes distordues (DWIA), $g(q)$ est une fonction plus compliquée qui tient compte des différentes distorsions. $S$ est le facteur spectroscopique. $(\mathrm{d} \sigma / \mathrm{d} \Omega)_{0, x}$ représente la section efficace de diffusion entre la particule incidente et l'agrégat $x$ hors de la couche d'énergie. L'approximation habituelle, consiste à la remplacer par la section efficace de diffusion libre, prise sur la couche d'énergie.

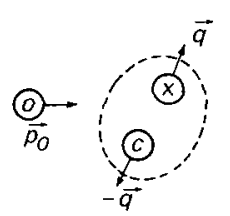

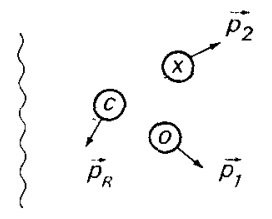<smiles>CC(N)C(C)(C)C</smiles>

FIG. 1. 
L'expérimentateur cherchera à obtenir des informations sur le spectre en énergie $E_{\mathrm{s}}$ et la distribution en impulsion q. Pour ce faire il travaillera, en général, en géométrie coplanaire et après avoir identifié les particules, mesurera en coïncidence, les énergies $E_{1}$ et $E_{2}$ pour différents couples d'angles $\theta_{1}$ et $\theta_{2}$. La distribution de la section efficace en fonction de l'impulsion sera établie soit à partir d'une distribution angulaire, soit d'une distribution en énergie, faite à un couple d'angle incluant la condition cinématique $p_{\mathrm{R}}=\mathbf{0}$.

On peut obtenir de ces mesures des renseignements sur le mécanisme de réaction et sur la structure en agrégat du noyau cible par la mesure des facteurs spectroscopiques.

Ainsi la forme de la distribution en impulsion, autour de la valeur $q=0$ est très caractéristique dans les réactions quasi élastiques. Suivant que le moment de l'agrégat dans le noyau est un état $L=0$ ou $L \neq 0$, la section efficace présentera un maximum ou un minimum pour $q=0$. C'est ce que l'on voit sur la figure 2 qui représente les distributions angulaires
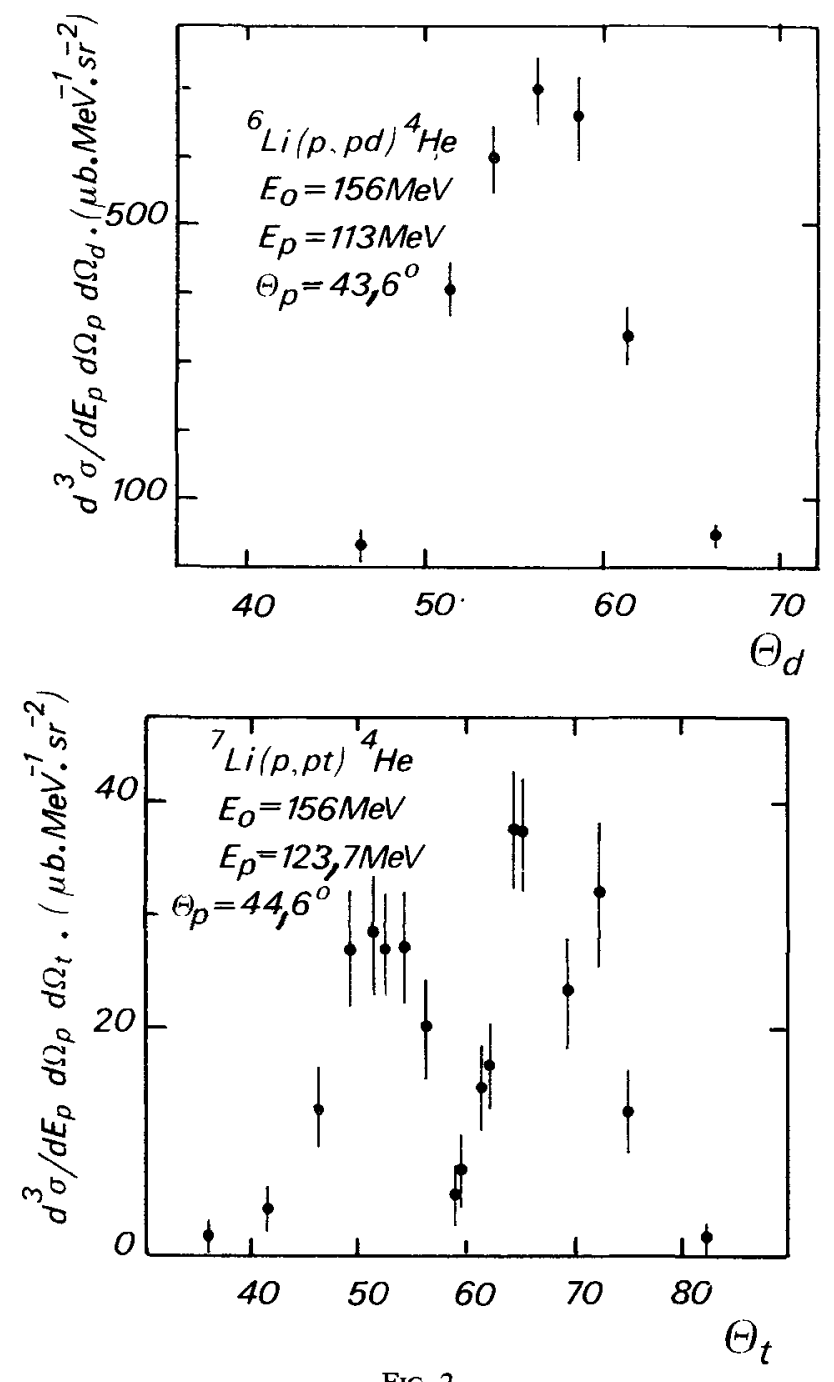

FIG. 2. obtenues à Orsay à $156 \mathrm{MeV}$ [4] pour les réactions ${ }^{6} \mathrm{Li}(\mathrm{p}, \mathrm{pd}){ }^{4} \mathrm{He}(L=0)$ et ${ }^{7} \mathrm{Li}(\mathrm{p}, \mathrm{pt})^{4} \mathrm{He}(L=1)$. En général la géométrie finie des détecteurs et plus particulièrement les effets de distorsion ont tendance à combler ces minima. Cependant les états $L=0$ sont assez faciles à identifier dans ces réactions.

En ce qui concerne le mécanisme de réaction, nous parlerons brièvement à titre d'exemple, d'une expérience $(\alpha, 2 \alpha)$ sur ${ }^{6} \mathrm{Li}$ faite à $42,8 \mathrm{MeV}$ par $\mathrm{P}$. Gaillard et al. [5]. Son but était de montrer l'influence de la section efficace de diffusion $(\alpha, \alpha)$ et de préciser si, dans l'approximation de la couche d'énergie, elle devait être prise à l'énergie avant réaction (forme $E_{i}$ ) ou après réaction (forme $E_{\mathrm{f}}$ ). Pour ce faire les auteurs ont utilisé l'importante résonance qui apparaît vers $20 \mathrm{MeV}$ centre de masse, dans la diffusion $\alpha-\alpha$. A l'énergie incidente de $42,8 \mathrm{MeV}$, cette résonance doit donner un minimum dans la section efficace $(\alpha, 2 \alpha)$ au voisinage de $q=0 \mathrm{MeV} / \mathrm{c}$ pour la forme $E_{\mathrm{f}}$ et de $q=+20 \mathrm{MeV} / \mathrm{c}$ pour la forme $E_{\mathrm{i}}$. Le résultat obtenu, que l'on voit sur la figure 3 montre de façon évidente l'influence de la diffusion $(\alpha-\alpha)$ et permet de déterminer sans ambiguité que celle-ci doit être considérée à l'énergie après réaction (forme $E_{\mathrm{f}}$ ).

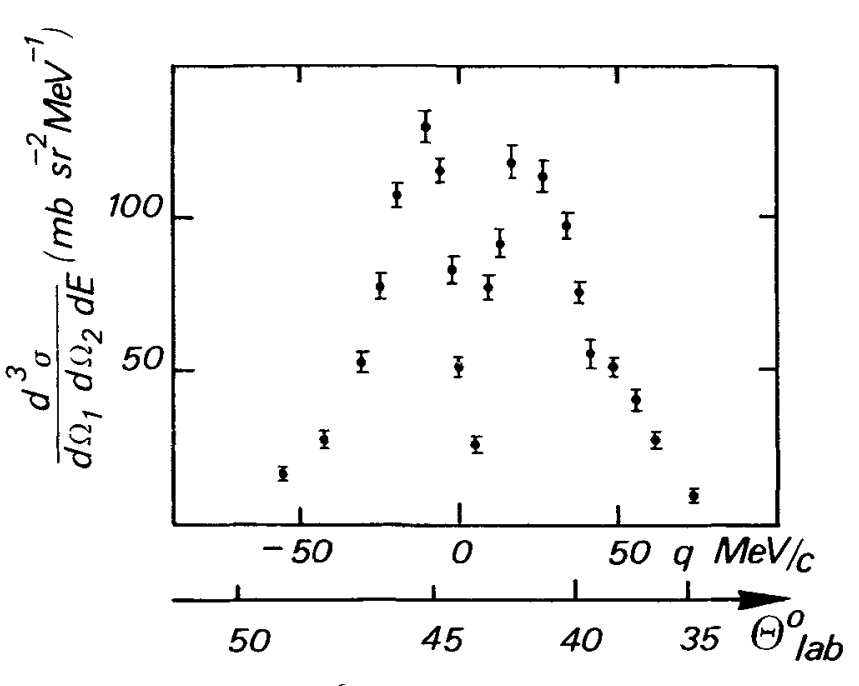

Fig. 3. $-{ }^{6} \mathrm{~L}(\alpha, 2 \alpha) \mathrm{d}$ à $42,8 \mathrm{MeV}$.

3. Evolution des expériences. - Au cours de ces dernières années, dans les différentes expériences qui ont été consacrées à ce sujet, on constate une évolution vers des mesures de plus en plus précises et sophistiquées. Il en est de même pour l'outil théorique qui permet de les interpréter.

Si dans l'ensemble, les particules incidentes les plus utilisées restent le proton et l'alpha et plus rarement l'électron, la mise en service de nouvelles machines comme les cyclotrons de Louvain-la-Neuve et de Maryland accélérant des protons jusqu'à $100 \mathrm{MeV}$, ont permis ou permettent d'envisager des expériences de diffusion quasi élastique avec une résolution suffisante pour séparer les niveaux des noyaux résiduels. Parallèlement ceci est rendu possible par de 
nouveaux types de détecteurs, comme ceux au germanium intrinsèque, qui permettent une mesure de l'énergie de protons jusqu'à $60-70 \mathrm{MeV}$ avec les mêmes qualités qu'une barrière de surface.

Une évolution apparaît aussi dans le choix des noyaux étudiés. Ceux-ci sont de plus en plus lourds et certaines tentatives ont été faites à Orsay jusqu'à ${ }^{232} \mathrm{Th}$ [6]. Si l'étude de la structure $\alpha$ reste un domaine de choix, des expériences ont été faites pour mettre en évidence et étudier d'autres agrégats. Enfin des faisceaux d'énergie élevée, comme ceux de Saturne, commencent à être utilisés.

Parallèlement l'outil théorique dont dispose l'expérimentateur a évolué. Il y a quelques années la PWIA était seule utilisée et les calculs que l'on pouvait faire avaient toutes les incertitudes et les défauts des ondes planes. Depuis ont été élaborés des programmes tenant compte des distorsions tout en restant dans l'approximation de l'impulsion (DWIA) [7, 8] et qui permettent d'extraire des facteurs spectroscopiques avec beaucoup plus de précision.

Les trois communications reçues sur ce sujet, s'inscrivent bien dans cette ligne d'évolution.

4. Réactions $(\mathbf{p}, \mathbf{p} \alpha)$ à $600 \mathrm{MeV}$. - Cette expérience, réalisée à Saturne par $\mathrm{R}$. Anne et al. [9] a porté sur les noyaux ${ }^{6} \mathrm{Li},{ }^{12} \mathrm{C},{ }^{24} \mathrm{Mg},{ }^{27} \mathrm{Al}$ et ${ }^{40} \mathrm{Ca}$. Une énergie incidente aussi élevée est intéressante car elle correspond bien au domaine de validité de l'approximation de l'impulsion et à cette énergie les distorsions doivent être minimes. Par ailleurs, les réactions parasites comme les processus séquentiels sont négligeables. Mais par contre, il est difficile d'obtenir une résolution suffisante pour séparer les états excités des noyaux résiduels.

Le dispositif expérimental comportait deux analyseurs magnétiques équipés des détecteurs classiques à cette énergie : chambres proportionnelles et scintillateurs. Une bonne identification des particules était assurée par la mesure de la perte d'énergie, de l'impulsion et du temps de vol ainsi que le montre la séparation ${ }^{3} \mathrm{He},{ }^{4} \mathrm{He}$. La résolution globale en énergie était d'environ $9 \mathrm{MeV}$.

La figure 4 donne un exemple des résultats obtenus pour les noyaux de ${ }^{6} \mathrm{Li}$ et de ${ }^{12} \mathrm{C}$. Le spectre en énergie de séparation montre clairement l'existence d'un pic de diffusion quasi élastique. Il en est d'ailleurs de même pour l'ensemble des noyaux à l'exception de ${ }^{27} \mathrm{Al}$. La distribution en moment de la figure 5 décroît bien aux valeurs élevées de $p_{R}$, ce qui indique une très faible contribution des réactions parasites.

5. Réactions $(p, p \alpha)$ à $157 \mathrm{MeV}$. - Dans cette expérience faite à Orsay par D. Bachelier et al. [10], les auteurs se sont intéressés aux noyaux pairs-pairs ${ }^{24} \mathrm{Mg},{ }^{28} \mathrm{Si},{ }^{40} \mathrm{Ca}$ et ${ }^{58} \mathrm{Ni}$. La résolution en énergie, de $2,6 \mathrm{MeV}$, était suffisante pour séparer les niveaux des noyaux résiduels.

La figure 5 représente le spectre en énergie d'excitation obtenu pour la réaction ${ }^{40} \mathrm{Ca}(\mathrm{p}, \mathrm{p} \alpha)^{36} \mathrm{Ar}$, pour
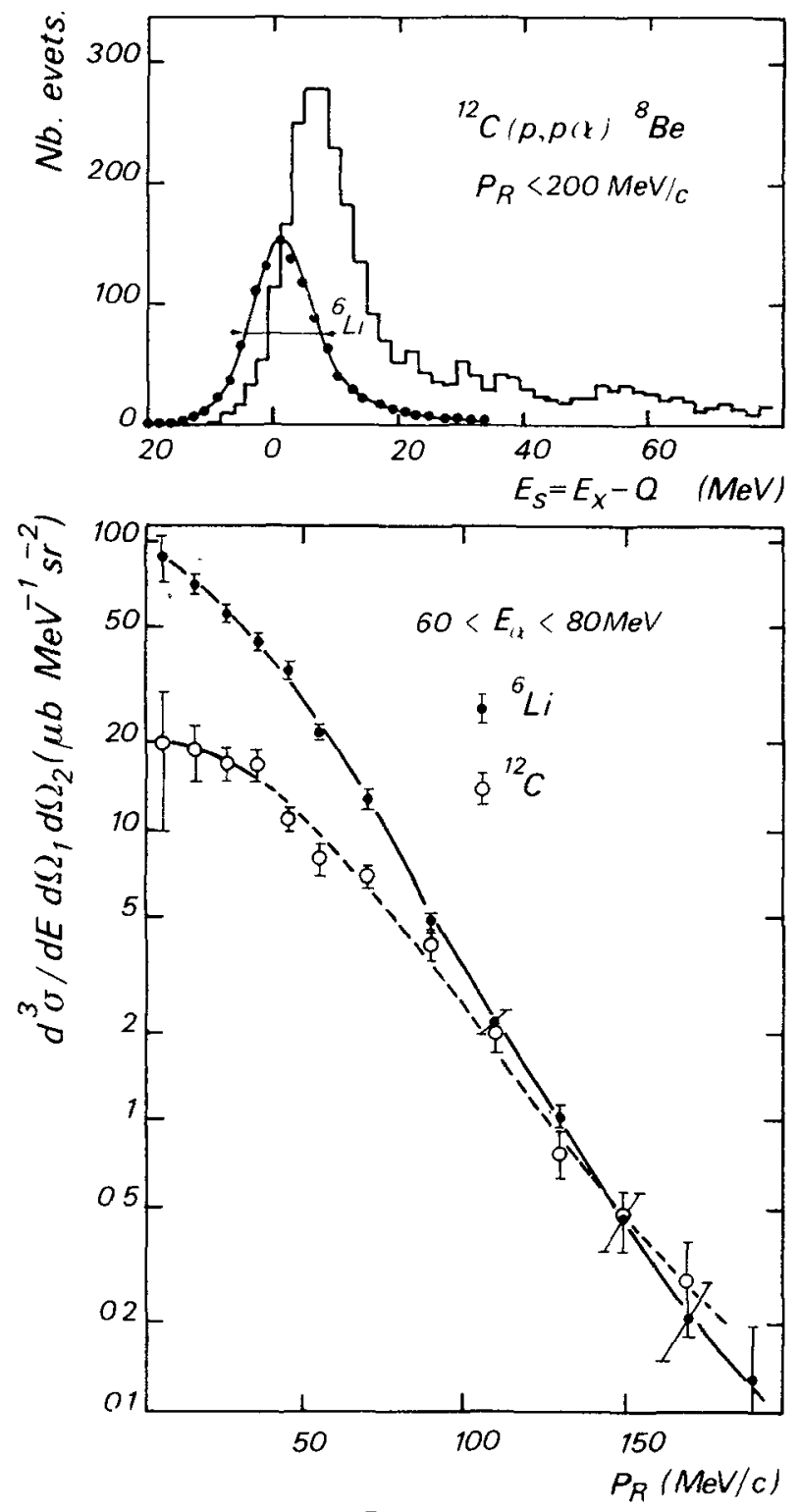

FIG. 4.

deux plages d'impulsion de recul. On voit nettement apparaître le fondamental $0^{+}$et le premier excité $2^{+}$, ainsi qu'un pic très marqué dans la région de $4,4 \mathrm{MeV}$. Le fait que ce pic soit très visible dans la région des grands moments de recul, laisse à penser qu'il s'agit du $4^{+}$. Les distributions en moments de ces trois niveaux sont représentées sur la figure 6 . La distribution du fondamental, qui présente un maximum marqué au voisinage de $q=0 \mathrm{MeV} / \mathrm{c}$ est tout à fait compatible avec un état $L=0$, auquel on doit s'attendre. En ce qui concerne les deux autres niveaux, la largeur des distributions est compatible avec des valeurs $L \neq 0$, les minima étant comblés par des effets de distorsion, qui semblent importants ici. Les auteurs signalent la faible valeur des sections efficaces pour ${ }^{58} \mathrm{Ni}$, de 5 à 15 fois plus petites que sur ${ }^{40} \mathrm{Ca}$. Cet effet de dépendance des sections efficaces avec la masse ne fait que confirmer les observations faites précédemment [6]. 


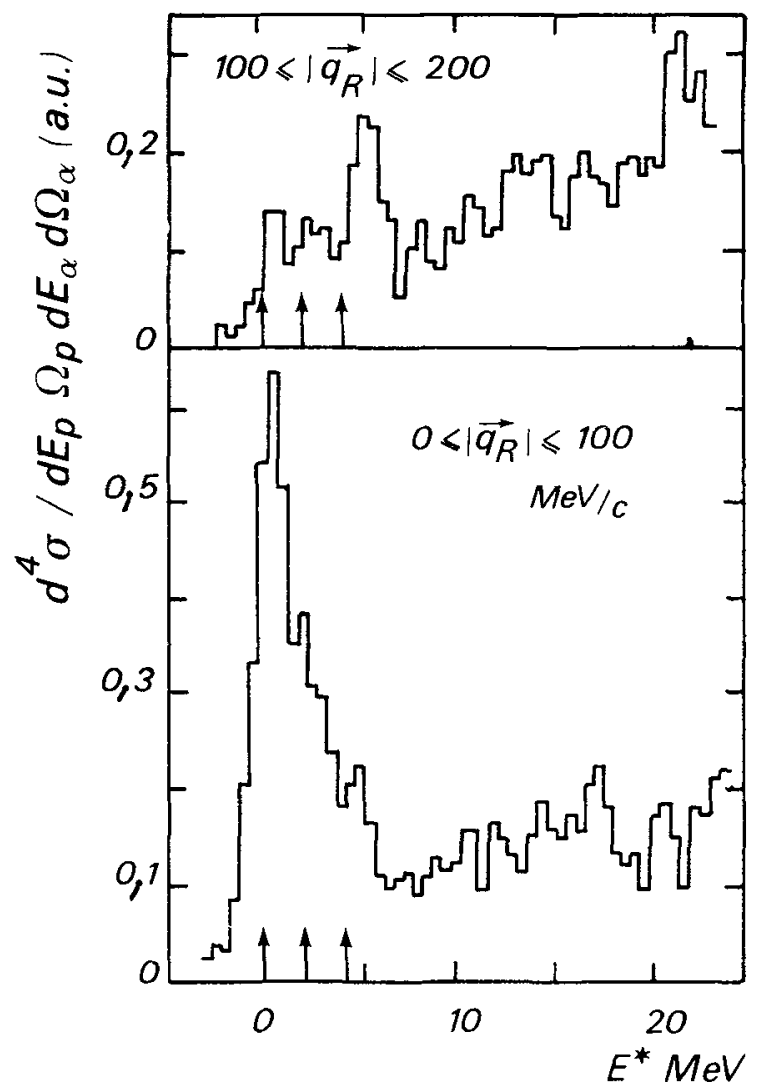

FIG. 5. - Spectre en énergie d'excitation du noyau de recul pour la réaction ${ }^{40} \mathrm{Ca}(\mathrm{p}, \mathrm{p} \alpha)^{36} \mathrm{Ar}$.

Des calculs en DWIA, faits avec le code de Chant [9] ont été comparés aux résultats expérimentaux et ont permis d'extraire les facteurs spectroscopiques $S_{\alpha}$ pour les états $0^{+}$et $2^{+}$des noyaux résiduels ${ }^{20} \mathrm{Ne}$; ${ }^{24} \mathrm{Mg}$ et ${ }^{36} \mathrm{Ar}$. Les résultats obtenus figurent dans le tableau I.

6. Réactions (p, pd), $(p, p t)$ et $\left(p, p{ }^{3} \mathrm{He}\right)$ à $75 \mathrm{MeV}$. - Dans cette expérience, effectuée au cyclotron de Louvain-la-Neuve, par Grossiord et al. [11], une étude de la structure en agrégats $\mathrm{d}, \mathrm{t},{ }^{3} \mathrm{He}$ de ${ }^{12} \mathrm{C}$ et ${ }^{16} \mathrm{O}$ a été tentée. De très rares expériences ont été faites sur ce type d'agrégats [4] pour des noyaux cibles plus lourds que le lithium, et les résolutions en énergie n'étaient pas suffisantes pour séparer les niveaux des noyaux résiduels. Dans cette expérience une résolution globale de $400 \mathrm{keV}$ a été obtenue et a permis cette séparation. Expérimentalement ceci a été rendu

TABLEAU I

\begin{tabular}{ccl} 
Réactions & Niveaux & \multicolumn{1}{c}{$S_{\alpha}$} \\
- & - & \\
${ }^{24} \mathrm{Mg}(\mathrm{p}, \mathrm{p} \alpha){ }^{20} \mathrm{Ne}$ & $0^{+}$ & $0,28 \pm 0,15$ \\
& $2^{+}$ & $3,3 \pm 3,3$ \\
${ }^{28} \mathrm{Si}(\mathrm{p}, \mathrm{p} \alpha)^{24} \mathrm{Mg}$ & $0^{+}$ & $0,27 \pm 0,14$ \\
& $2^{+}$ & $1,0 \pm 1,0$ \\
${ }^{40} \mathrm{Ca}(\mathrm{p}, \mathrm{p} \alpha)^{36} \mathrm{Ar}$ & $0^{+}$ & $0,53 \pm 0,15$ \\
& $2^{+}$ & $2,9 \pm 2,9$
\end{tabular}

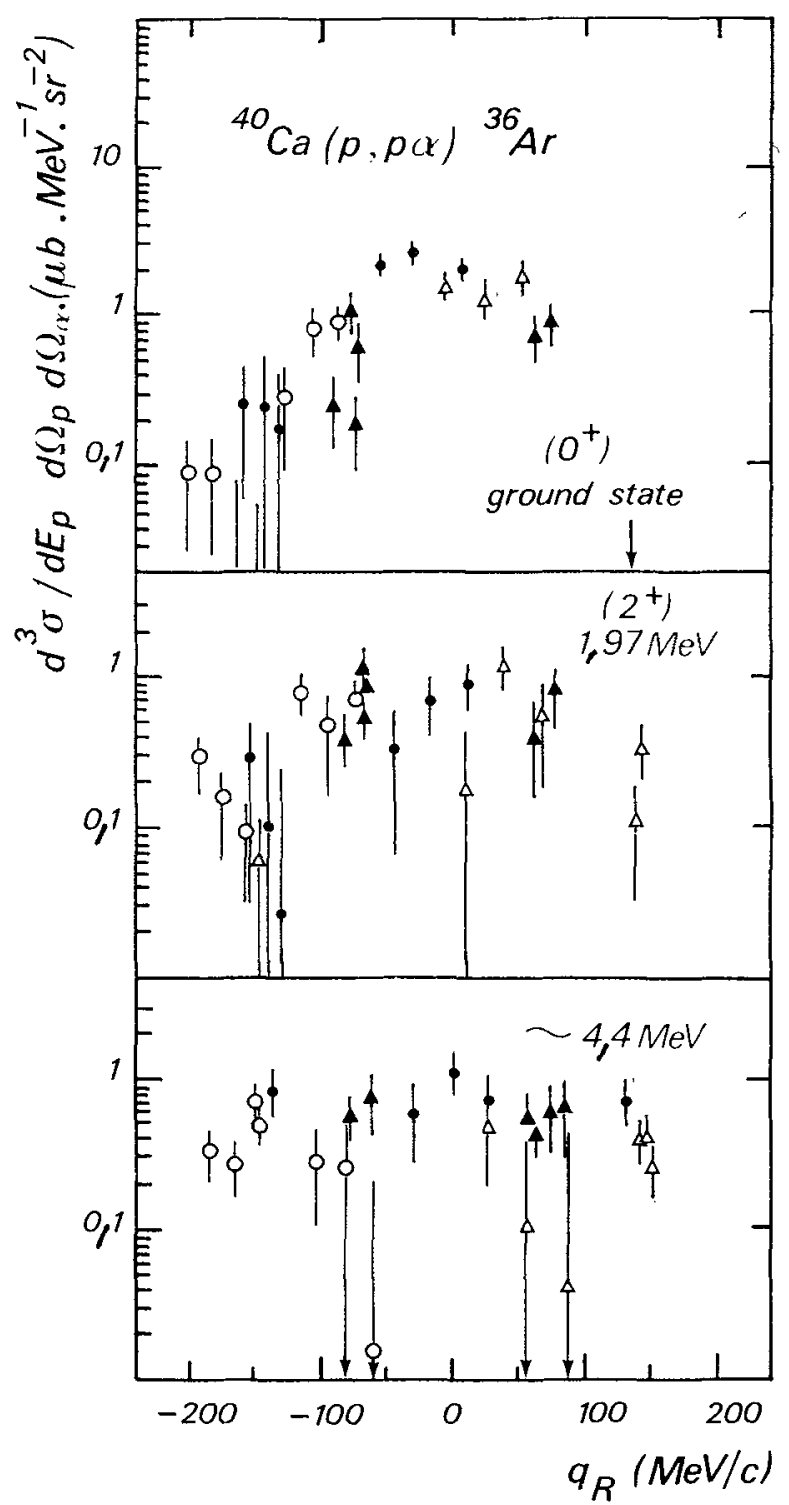

FIG. 6.

possible par l'utilisation d'un détecteur germanium intrinsèque de $7 \mathrm{~mm}$ pouvant détecter des protons de $50 \mathrm{MeV}$ avec une résolution de $40 \mathrm{keV}$.

La figure 7 montre le spectre d'excitation obtenu pour les réactions ${ }^{12} \mathrm{C}(\mathrm{p}, \mathrm{pd}){ }^{10} \mathrm{~B}$ et ${ }^{16} \mathrm{O}(\mathrm{p}, \mathrm{pd}){ }^{14} \mathrm{~N}$. Si le fondamental de ${ }^{10} \mathrm{~B}\left(3^{+}\right)$et les premiers niveaux, $1^{+} \mathrm{de}^{10} \mathrm{~B}$ et ${ }^{14} \mathrm{~N}$ sont bien peuplés, on voit apparaitre très nettement les niveaux $0^{+} \mathrm{T}=1$ de ${ }^{10} \mathrm{~B}$ et ${ }^{14} \mathrm{~N}$. Ceci est intéressant, car le fait qu'ils soient interdits dans un mécanisme de diffusion quasi élastique, amène à imaginer d'autres processus comme par exemple ceux décrits par les graphes de la figure 8 .

Le même problème se pose pour les réactions $(p, p t)$ et $\left(p, p{ }^{3} \mathrm{He}\right)$ sur l'oxygène où l'on observe l'excitation des niveaux de parité positive $\frac{1}{2}^{+}$. Un des processus pouvant être envisagé est, comme dans le cas précédent, une diffusion dans l'état final du proton sur le noyau résiduel. 


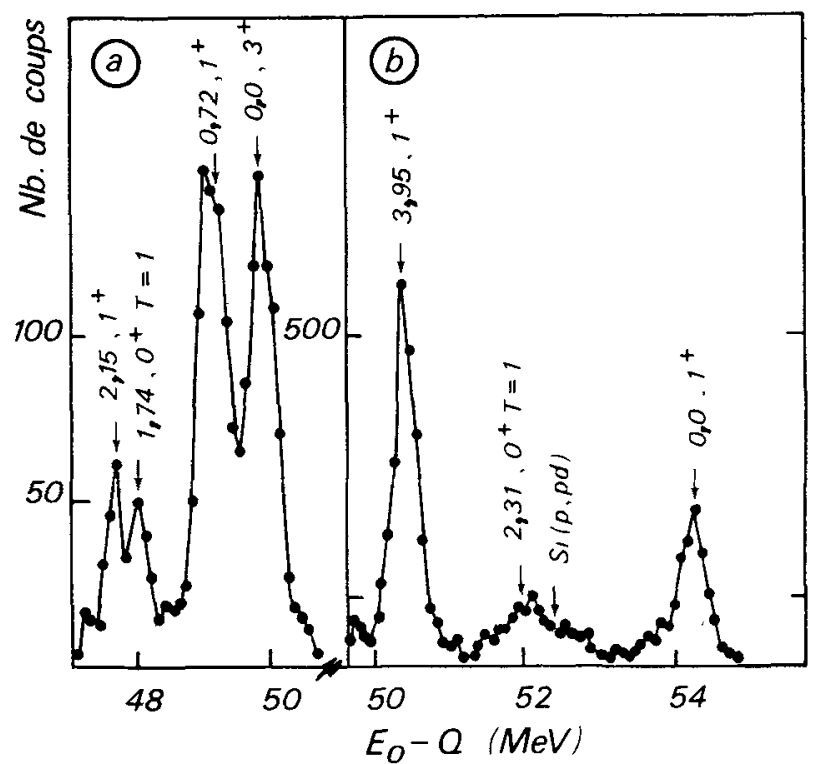

FIG. 7. - Spectre en énergie d'excitation du noyau de recul pour les réactions : a) $\left.{ }^{12} \mathrm{C}(\mathrm{p}, \mathrm{pd}){ }^{10} \mathrm{~B} ; b\right){ }^{16} \mathrm{O}(\mathrm{p}, \mathrm{pd})^{14} \mathrm{~N}$.

(a)
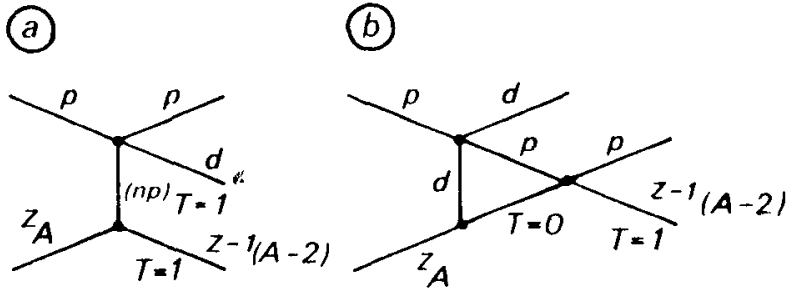

FIG. 8.
Si aucun calcul quantitatif n'a été effectué, par contre une comparaison relative des facteurs spectroscopiques entre niveaux conduisant aux mêmes valeurs de $L$, a été faite avec ceux calculés par Cohen et Kurath [12] et Kurath et Millener [13] ; un bon accord est obtenu ainsi que l'on peut le voir dans les tableaux qui figurent dans la communication de Grossiord et al. [11].

7. Conclusion. - Malgré toutes les expériences qui ont été effectuées jusqu'à maintenant dans le domaine des réactions de diffusion quasi élastique, il est certain que notre compréhension de la structure en agrégats des noyaux est loin d'être parfaite. Au demeurant peut-on parler d'agrégats pré-existants dans les noyaux, à plus forte raison lorsque ceux-ci sont de plus en plus lourds ? Il semble qu'aujourd'hui les opinions soient très partagées sur ce sujet. L'image que représente la figure 1 est certainement beaucoup trop simpliste et nous avons vu que lorsque les expériences s'affinent d'autres problèmes surgissent en particulier des problèmes de mécanismes de réaction.

L'utilisation relativement récente de la DWIA devrait permettre une étude approfondie de la spectroscopie. Une comparaison serait alors possible avec les renseignements que l'on obtient à partir des réactions de stripping et de pick-up. Pour cela il faut disposer d'expériences précises, faites avec une résolution en énergie suffisante, portant sur différents types d'agrégats et qui utilisent des projectiles de nature et d'énergie variées. De la même manière un soin particulier doit être apporté à l'étude des mécanismes de réaction.

Bibliographie

[1] Ruhla, C. et al., Phys. Lett. 2 (1962) 44. Rrou, M., Rev. Mod. Phys. 37 (1965) 375.

[2] James, A. N., Pugh, H. G., Nucl. Phys. 42 (1963) 44.

[3] IGo, G. et al., Phys. Rev. 131 (1963) 337.

[4] BACHelier, D., Thèse Doct. ès-Sci. Phys., Paris/Orsay (1971).

[5] Gall lard, P. et al., Phys. Rev. Lett. 25 (1970) 593.

[6] BaChelier, D. et al., Phys. Rev. C 7 (1973) 165.

[7] J J AIN, A. K., Ph. D. Thesis (1972), Univ. of Bombay, Report BARC-635.

[8] Chant, N. S., Technical report (en préparation), Univ. of Maryland.
Chant, N. S. and Woody, R., 1973 Progress Report, Cyclotron Lab., Univ. of Maryland.

[9] ANNE, R. et al., Communication présentée au Congrès de la Société Française de Physique, Dijon (1975).

[10] BACHELIER, D. et al., Communication présentée au Congrès de la Société Française de Physique, Dijon (1975).

[11] Grossiord, J. Y. et al., Communication présentée au Congrès de la Société Française de Physique, Dijon (1975).

[12] Cohen, L. and Kurath, D., Nucl. Phys. A 141 (1970) 145.

[13] Kurath, D. and Millener, D. J., Nucl. Phys. A 238 (1975) 269. 